\title{
Molecular identification of symbiotic dinoflagellates in Pacific corals in the genus Pocillopora
}

\author{
H. Magalon · J.-F. Flot · E. Baudry
}

Published online: 21 August 2008

(C) Springer-Verlag 2008

\section{Erratum to: Coral Reefs (2007) DOI 10.1007/s00338-007-0215-0}

The authors regret that they made an error in reporting the primers used to amplify and sequence the ITS1 region, mentioned in the Material and methods section. The primer ITSint-rev was incorrectly reported and an internal primer which was incorrectly omitted has been added below.

\section{Materials and methods}

PCR amplifications of zooxanthellae nrDNA

[The second paragraph of this section should be replaced with the following text]

To investigate zooxanthella diversity in more detail, the Internal Transcribed Spacer 1 region (ITS1), which is a non-coding region that evolves faster than the $28 \mathrm{~S}$ nrDNA

The online version of the original article can be found under doi: 10.1007/s00338-007-0215-0.

\section{H. Magalon ( $\square)$}

UMR CNRS 7625, Laboratoire Fonctionnement et Evolution des Systèmes Ecologiques, Université Pierre et Marie Curie,

7 quai saint Bernard, 75005 Paris, France

e-mail: hmagalon@snv.jussieu.fr

J.-F. Flot

UMR UPMC-CNRS-MNHN-IRD 7138, Département Systématique et Evolution, Muséum National d'Histoire Naturelle, 43 rue Cuvier, 75005 Paris, France

E. Baudry

UMR CNRS 8079, Laboratoire Ecologie, Systématique et Evolution, Université Paris-Sud, Bat 362, 91405 Orsay Cedex, France
(Santos et al. 2002a), was sequenced. Two primers were used to amplify both ITS1 and ITS2: one designed in the $3^{\prime}$-end of the 18S DNA [ITS-for: $5^{\prime}$-CGG TGA ATT ATT CGG ACT GAC-3'; reverse of SYM3, modified from Hunter et al. (1997)] and the other in the $5^{\prime}$-end of the 28S DNA [ITS-rev: 5'-TCC TCC GCT TAT TGA TAT GC-3' from Hunter et al. (1997)]. The PCR protocol was identical to that of $28 \mathrm{~S}$ nrDNA amplification, except amplifications were carried out in a total volume of $30 \mu \mathrm{l}$. Amplified products were purified using QIAquick PCR purification kit (Qiagen) following the manufacturer's instructions and ITS1 region was directly sequenced in both directions using 3.2 pmol of the primer ITS-for and an internal primer in the $5.8 \mathrm{~S}$ region [ITSint-rev: $5^{\prime}$-CAC GGA GTT CTG CAA TTC- $3^{\prime}$ (reverse of ITSintfor2 from LaJeunesse and Trench (2000))]. [The region ITS2 can be sequenced using ITS-rev and ITSintfor 2 from LaJeunesse and Trench (2000).] Reagents and cycling conditions were as specified in the ABI Prism Big Dye Terminator Cycle Sequencing Ready Reaction kit (PE Applied Biosystems, Foster City, California). 